\title{
HOMOGENEOUS FOURIER MULTIPLIERS IN THE PLANE
}

\author{
JAVIER DUOANDIKOETXEA AND ADELA MOYUA
}

(Communicated by J. Marshall Ash)

\begin{abstract}
Given a homogeneous of degree zero function on the plane, we study conditions on the first derivative of its restriction to the unit circle in order to deduce that it is an $L^{p}$-multiplier.
\end{abstract}

\section{INTRODUCTION}

Let $m$ be a bounded function in $\mathbb{R}^{2}$ and $T$ the operator given by $(T f)^{\wedge}=$ $m \hat{f}, \forall f \in L^{2}$. We say that $m$ is an $L^{p}$-multiplier $\left(m \in \mathscr{M}_{p}\right)$ if $T$ can be extended as a bounded operator to $L^{p}\left(\mathbb{R}^{2}\right)$. It is well known that $m \in \mathscr{M}_{2}$ if and only if $m$ is bounded and that $m \in \mathscr{M}_{1}$ if and only if the Fourier transform of $m$ is a finite Borel measure. For all other values of $p$ no simple characterizations exist.

The celebrated Marcinkiewicz multiplier theorem gives a sufficient condition on $m$ in order to get an $L^{p}$-multiplier. When $n=2$ the condition is the following [6, p. 109]:

Let $m$ be a bounded function, continuous together with its partial derivatives up to order 2 on each quadrant and such that

$$
\begin{aligned}
& \sup _{j \in \mathbb{Z}} \sup _{s \in \mathbb{R}} \int_{I_{j}}\left|\frac{\partial m}{\partial t}(t, s)\right| d t<+\infty, \\
& \sup _{j \in \mathbb{Z}} \sup _{t \in \mathbb{R}} \int_{I_{j}}\left|\frac{\partial m}{\partial s}(t, s)\right| d s<+\infty, \\
& \sup _{i, j \in \mathbb{Z}} \int_{I_{i} \times I_{j}}\left|\frac{\partial^{2} m}{\partial t \partial s}(t, s)\right| d t d s<+\infty
\end{aligned}
$$

then $m \in \mathscr{M}_{p}, 1<p<\infty$.

Here $I_{i}, I_{j}$ are dyadic intervals in $\mathbb{R}$, that is, intervals of the form $\left[2^{j}, 2^{j+1}\right]$ or $\left[-2^{j+1},-2^{j}\right]$.

In many interesting cases $m$ is a homogeneous function (of degree zero because it is bounded) and, using the same letter $m$ to denote its restriction to

Received by the editors October 6, 1989 and, in revised form, June 21, 1990.

1980 Mathematics Subject Classification (1985 Revision). Primary 42B15. 
the unit circle, the above conditions are equivalent to

$$
\begin{aligned}
& \sup _{j \in \mathbb{Z}} \int_{I_{j}}\left|m^{\prime}(\theta)\right| d \theta<+\infty, \\
& \sup _{j \in \mathbb{Z}} \int_{I_{j}}\left|\theta m^{\prime \prime}(\theta)\right| d \theta<+\infty,
\end{aligned}
$$

where $I_{j}$ is the dyadic $\operatorname{arc}$ on $S^{1}$ given by $\left[\operatorname{arctg} 2^{j}, \operatorname{arctg} 2^{j+1}\right.$ ], plus similar conditions after rotations of $\pi / 2, \pi$, and $3 \pi / 2$.

Because homogeneous multipliers are given by their restrictions to the unit circle, they are in some sense one-dimensional. It seems natural to ask whether as in the one-dimensional Marcinkiewicz theorem, conditions on the first derivative of $m$ will be enough to assure that $m \in \mathscr{M}_{p}, 1<p<\infty$. This question was posed to us by José $L$. Rubio de Francia and this paper tries to answer it.

We can concentrate ourselves on multipliers supported on $[0, \pi / 4]$. The main singularity on $m$ will appear near the positive $O X$ axis but the results trivially extend to the case when several singular directions appear. Our main theorem is the following

Theorem 1. Let $m$ be a bounded function on $S^{1}$, supported on $[0, \pi / 4]$ and absolutely continuous on each dyadic interval $I_{j}=\left\{\theta / 2^{-j} \frac{\pi}{4}<\theta<2^{-j+1} \frac{\pi}{4}\right\}$ and such that

$$
\sup _{j \in \mathbb{N}} \int_{I_{j}}\left|\theta m^{\prime}(\theta)\right|^{q} d \theta / \theta<+\infty
$$

for some $q$, then the homogeneous (of degree zero) extension of $m$ is an $L^{p}$ multiplier: (i) for $1<p<\infty$ if $q \geq 2$; (ii) for $\left|\frac{1}{p}-\frac{1}{2}\right|<\frac{3}{4}-\frac{1}{2 q}$ if $1<q<2$.

In particular, the typical condition for one-dimensional multipliers $\left|m^{\prime}(\theta)\right| \leq$ $C|\theta|^{-1}$ is enough to get an $L^{p}$-multiplier, $1<p<\infty$, if $m$ is bounded.

When the restriction of the multiplier to the interval $I_{j}$ is obtained from a fixed one by dilation, then we have a better result:

Theorem 2. Let $m_{0}$ be an absolutely continuous function on $[\pi / 8, \pi / 4]$, extended by zero outside this interval then $m(\theta)=\sum_{j=0}^{\infty} m_{0}\left(2^{j} \theta\right), 0<\theta<\pi / 4$, defines an $L^{p}$-multiplier $1<p<\infty$.

In the proofs of these theorems we will use a Littlewood-Paley function associated to a decomposition of the plane into angular sectors of lacunary aperture:

Let $I$ be a given interval contained in $[\pi / 8, \pi / 4]$ and $S_{j}$ the multiplier operator given by

$$
\left(S_{j} f\right)^{\wedge}(\xi)=\chi_{I}\left(2^{j} \theta\right) \hat{f}(\xi), \quad j \geq 0, \theta=\operatorname{arctg} \xi_{2} / \xi_{1},
$$

where $\chi_{I}$ is the characteristic function of $I$. Then, there exists a constant $C_{p}$ 
depending only on $p$ and not on $I$, such that

$$
\left\|\left(\sum_{j}\left|S_{j} f\right|^{2}\right)^{1 / 2}\right\|_{p} \leq C_{p}\|f\|_{p}, \quad 1<p<\infty .
$$

Also, if $\theta_{j}$ is a lacunary set of directions on $S^{1}$ and $M_{j}$ are their associated directional Hardy-Littlewood maximal functions, that is,

$$
M_{j} f(x)=\sup _{h>0} \frac{1}{2 h} \int_{-h}^{h}\left|f\left(x-r e^{i \theta_{j}}\right)\right| d r,
$$

we have

$$
\left\|\sup _{j} M_{j} f\right\|_{p} \leq C_{p}\|f\|_{p}, \quad 1<p<\infty .
$$

Both results are in [5]. The use of Littlewood-Paley inequalities in order to get multiplier theorems is classical. What is probably new is the use of this angular decomposition of $\mathbb{R}^{2}$.

\section{PROOF OF THEOREM 1}

Let $I_{j}=\left[\theta_{j}, \theta_{j-1}\right)$ and $m_{j}=m \chi_{I_{j}}$. We can assume that $m_{j}$ is continuous from the right at $\theta_{j}$. Then if $\theta_{j}<\theta<\theta_{j-1}$,

$$
\begin{aligned}
m_{j}(\theta) & =m_{j}\left(\theta_{j}\right)+\int_{\theta_{j}}^{\theta} m^{\prime}(t) d t \\
& =m_{j}\left(\theta_{j}\right)+\int_{\theta_{j}}^{\theta_{j-1}} m^{\prime}(t) \chi_{\left[t, \theta_{j-1}\right)}(\theta) d t
\end{aligned}
$$

so that, denoting by $S_{j}$ the operator $\left(S_{j} f\right)^{\wedge}=\chi_{I_{j}} \hat{f}$,

$$
\begin{aligned}
T f & =\sum_{j} T S_{j} f \\
& =\sum_{j}\left[m_{j}\left(\theta_{j}\right) S_{j} f+\int_{\theta_{j}}^{\theta_{j-1}} m^{\prime}(t) H_{t} S_{j} f d t\right],
\end{aligned}
$$

where $H_{t}$ is the multiplier operator defined by the characteristic function of the semiplane determined by the line through the origin with direction $t$. The contribution of the first terms in the sum is easy to handle

$$
\begin{aligned}
\left\|\sum_{j} S_{j}\left(m_{j}\left(\theta_{j}\right) S_{j} f\right)\right\|_{p} & \leq C_{p}\left\|\left(\sum_{j}\left|m_{j}\left(\theta_{j}\right) S_{j} f\right|^{2}\right)^{1 / 2}\right\|_{p} \\
& \leq C_{p}^{\prime}\left\|\left(\sum_{j}\left|S_{j} f\right|^{2}\right)^{1 / 2}\right\|_{p} \leq C_{p}^{\prime \prime}\|f\|_{p},
\end{aligned}
$$


where the third and first inequalities come from (2) and its dual and the second one is a consequence of the boundedness of $m$.

So let

$$
T_{j} f(x)=\int_{\theta_{j}}^{\theta_{j-1}} m^{\prime}(t) H_{t} S_{j} f(x) d t .
$$

Since $T_{j} f=T_{j} S_{j} f$ applying again the inequality dual to (2)

$$
\left\|\sum_{j} T_{j} f\right\|_{p}=\left\|\sum_{j} T_{j} S_{j} f\right\|_{p} \leq C_{p}\left\|\left(\sum_{j}\left|T_{j} f\right|^{2}\right)^{1 / 2}\right\|_{p} .
$$

Case $q=2$. The Cauchy-Schwarz inequality gives

$$
\begin{aligned}
\left|T_{j} f(x)\right|^{2} & \leq\left(\int_{\theta_{j}}^{\theta_{j-1}}\left|t^{\prime}(t)\right|^{2} \frac{d t}{t}\right)\left(\int_{\theta_{j}}^{\theta_{j-1}}\left|H_{t} S_{j} f(x)\right|^{2} \frac{d t}{t}\right) \\
& \leq C \int_{\pi / 8}^{\pi / 4}\left|H_{2^{-j}} S_{j} f(x)\right|^{2} d t
\end{aligned}
$$

after a change of variable. For a fixed $t$ in $[\pi / 8, \pi / 4],\left\{H_{2^{-j} t} S_{j}\right\}$ is a sequence of operators associated to the lacunary sequence of sectors obtained by dilation of $[t, \pi / 4]$. Applying (2) we have

$$
\left\|\left(\sum_{j}\left|H_{2^{-j} t} S_{j} f\right|^{2}\right)^{1 / 2}\right\|_{p} \leq C_{p}\|f\|_{p}
$$

with $C_{p}$ independent on $t$ and the result follows.

Case $1<q<2$. We will prove the following inequality:

Given $u \in L^{r}\left(\mathbb{R}^{2}\right)$, there exists $U \in L^{r}\left(\mathbb{R}^{2}\right)$ such that $\|U\|_{r} \leq C\|u\|_{r}$ and

$$
\int\left|T_{j} f(x)\right|^{2} u(x) d x \leq C \int\left|S_{j} f(x)\right|^{2} U(x) d x
$$

with a constant uniform in $j$, for $\frac{1}{r}<\frac{3}{2}-\frac{1}{q}$.

When $p \geq 2$ satisfies $\frac{1}{2}-\frac{1}{p}<\frac{3}{4}-\frac{1}{2 q}$, the exponent $r$ conjugate to $\frac{p}{2}$ satisfies $\frac{1}{r}<\frac{3}{2}-\frac{1}{q}$. The above claim implies then

$$
\left\|\left(\sum_{j}\left|T_{j} f\right|^{2}\right)^{1 / 2}\right\|_{p} \leq C_{p}\left\|\left(\sum_{j}\left|S_{j} f\right|^{2}\right)^{1 / 2}\right\|_{p} .
$$

In order to prove (4) we apply the Cauchy-Schwarz inequality to get

$$
\left|T_{j} f(x)\right|^{2} \leq\left(\int_{\theta_{j}}^{\theta_{j-1}}\left|t m^{\prime}(t)\right|^{q} \frac{d t}{t}\right)\left(\int_{\theta_{j}}^{\theta_{j-1}}\left|t m^{\prime}(t)\right|^{2-q}\left|H_{t} S_{j} f(x)\right|^{2} \frac{d t}{t}\right)
$$


then,

$$
\begin{aligned}
\int\left|T_{j} f(x)\right|^{2} u(x) d x & \leq C \int_{\theta_{j}}^{\theta_{j-1}}\left|\operatorname{tm}^{\prime}(t)\right|^{2-q} \int_{\mathbb{R}^{2}}\left|H_{t} S_{j} f(x)\right|^{2} u(x) d x \frac{d t}{t} \\
& \leq C \int_{\theta_{j}}^{\theta_{j-1}}\left|\operatorname{tm}^{\prime}(t)\right|^{2-q} \int_{\mathbb{R}^{2}}\left|S_{j} f(x)\right|^{2}\left(M_{t} u^{\delta}(x)\right)^{1 / \delta} d x \frac{d t}{t}
\end{aligned}
$$

with $\delta>1$. Here $M_{t}$ is the maximal function along the direction orthogonal to $t$ so that $\left(M_{t} u^{\delta}\right)^{1 / \delta}$ is an $A_{1}$-weight for $H_{t}$ (see [4]). We apply Hölder's inequality to the integral in $t$ with exponents $q /(2-q)$ and $q /(2 q-2)=q^{\prime} / 2$ so that

$$
\int\left|T_{j} f(x)\right|^{2} u(x) d x \leq C \int_{\mathbb{R}^{2}}\left|S_{j} f(x)\right|^{2}\left(\int_{\theta_{j}}^{\theta_{j-1}}\left(M_{t} u^{\delta}(x)\right)^{\frac{1}{\delta} \frac{q^{\prime}}{2}} \frac{d t}{t}\right)^{2 / q^{\prime}} d x .
$$

We can take

$$
\begin{aligned}
U(x) & =\sup _{j}\left(\int_{\theta_{j}}^{\theta_{j-1}}\left(M_{t} u^{\delta}(x)\right)^{\frac{1}{\delta}} \frac{q^{\prime}}{2} \frac{d t}{t}\right)^{2 / q^{\prime}} \\
& =\sup _{j}\left(\int_{\pi / 8}^{\pi / 4}\left(M_{2^{-j} t} u^{\delta}(x)\right)^{\frac{1}{\delta}} \frac{q^{\prime}}{2} \frac{d t}{t}\right)^{2 / q^{\prime}} .
\end{aligned}
$$

The inequality (4) and the restriction on the values of $r$ are now a consequence of the following lemma.

Lemma 3. The inequality

$$
\left(\int_{\mathbb{R}^{2}}\left(\int_{\pi / 8}^{\pi / 4} \sup _{j}\left|M_{2^{-j_{\theta}}} f(x)\right|^{s} d \theta\right)^{r / s} d x\right)^{1 / r} \leq C\|f\|_{r}
$$

holds if $\frac{1}{r}<\frac{1}{2 s}+\frac{1}{2}$.

For a fixed $\theta$ it corresponds to inequality (3). For a fixed $j$ it has been proved in [1] with the same restriction on $r$ (which is sharp). The proof of this lemma is a combination of the proofs of both results. Notice that when $r=s$ (hence also when $r>s$ ) the result is a consequence of (3) since the order of integration can be reversed. It is then enough to prove the lemma for $r=2$, $s<\infty$ and interpolate.

Sketch of the proof of the lemma. Let $\Phi \in C_{0}^{\infty}\left(\mathbb{R}^{2}\right), \psi \in C_{0}^{\infty}(\mathbb{R})$ be nonnegative functions with integral $1, \Phi$ radial, and $\psi(0) \neq 0$ and define

$$
\psi_{k}(s)=2^{-k} \psi\left(2^{-k} s\right), \quad \Phi_{k}(x)=2^{-2 k} \Phi\left(2^{-k} x\right) .
$$

Clearly, if $f \geq 0$

$$
M_{\theta} f(x) \leq C \sup _{k \in \mathbb{Z}} \int_{\mathbb{R}} f\left(x-s e^{i \theta}\right) \psi_{k}(s) d s .
$$


Since $\sup _{k, j} \iint f\left(y-s e^{i 2^{-j} \theta}\right) \psi_{k}(s) \Phi_{k-j}(x-y) d s d y$ is bounded by the strong maximal function in $\mathbb{R}^{2}$, we only need to consider $\sup _{k, j} A_{k, j} f(x, \theta)$ where $A_{k, j}$ is defined by

$$
A_{k, j} f(x, \theta)=\iint f\left(y-s e^{i 2^{-j} \theta}\right) \psi_{k}(s)\left(\delta-\Phi_{k-j}\right)(x-y) d s d y
$$

( $\delta$ is the Dirac mass at the origin), so that

$$
\left(A_{k, j} f(\cdot, \theta)\right)^{\wedge}(\xi)=\hat{\psi}\left(\left\langle 2^{k} \xi, e^{i 2^{-j} \theta}\right\rangle\right)\left(1-\widehat{\Phi}\left(2^{k-j} \xi\right)\right) \hat{f}(\xi) .
$$

This multiplier has good decay properties except when $\xi$ is close to the direction orthogonal to $e^{i 2^{-j} \theta}$. When $\theta$ moves between $\pi / 8$ and $\pi / 4$ the set of bad directions sweeps a sector of amplitude $2^{-j} \pi / 8$, disjoint for different values of $j$. Adapted to these sectors we define a sequence $\left\{\omega_{j}\right\}$ of homogeneous functions of degree zero, $C^{\infty}$ and such that $\omega_{j}$ is 1 on the $j$ th angle and its two adjacents and vanishes outside the union of the $j$ th angle and its four adjacents. Decompose $A_{k, j} f$ as the sum of $A_{k, j}^{1} f$ and $A_{k, j}^{2} f$ given by

$$
\begin{aligned}
& \left(A_{k, j}^{1} f\right)^{\wedge}(\xi, \theta)=\hat{\psi}\left(\left\langle 2^{k} \xi, e^{i 2^{-j} \theta}\right\rangle\right)\left(1-\widehat{\Phi}\left(2^{k-j} \xi\right)\right) \omega_{j}(\xi) \hat{f}(\xi), \\
& \left(A_{k, j}^{2} f\right)^{\wedge}(\xi, \theta)=\hat{\psi}\left(\left\langle 2^{k} \xi, e^{i 2^{-j} \theta}\right\rangle\right)\left(1-\widehat{\Phi}\left(2^{k-j} \xi\right)\right)\left(1-\omega_{j}(\xi)\right) \hat{f}(\xi) .
\end{aligned}
$$

Reasoning as in [5]

$$
\left(A_{k, j}^{2} f\right)(x, \theta) \leq C M_{S} f(x),
$$

where $M_{S}$ is the strong maximal function in $\mathbb{R}^{2}$. Then we only need to prove the lemma for $\sup _{k, j} A_{k, j}^{1} f(x)$. Following [1] we can prove instead

$$
\left\|\left(\sum_{j, k}\left|A_{k, j}^{1} f(x, \theta)\right|^{2}\right)^{1 / 2}\right\|_{L^{2}\left(L_{\beta}^{2}\right)} \leq C\|f\|_{2} \quad \forall \beta<\frac{1}{2},
$$

where $L_{\beta}^{2}$ is the Sobolev space on $S^{1}$ (see [6]). Since for all $s<\infty$ there exists $\beta<\frac{1}{2}$ such that $L_{\beta}^{2} \subset L^{s}$ (Sobolev embedding theorem), the lemma follows. In order to prove (5) one repeats the reasoning of [1] and uses the finite overlapping of the $\left\{\omega_{j}\right\}$ for the sum in $j$.

\section{Proof of TheOREM 2}

Going back to the decomposition $T f(x) \simeq \sum_{j} T_{j} f(x)$ we have

$$
\begin{aligned}
T_{j} f(x) & =\int_{\theta_{j}}^{\theta_{j-1}} m^{\prime}(t) H_{t} S_{j} f(x) d t \\
& =\int_{\pi / 8}^{\pi / 4} 2^{-j} m^{\prime}\left(2^{-j} t\right) H_{2^{-j} t} S_{j} f(x) d t
\end{aligned}
$$


and since $m(\theta)=\sum_{j=0}^{\infty} m_{0}\left(2^{j} \theta\right)$, when $\frac{\pi}{8}<\theta<\frac{\pi}{4}$ we have $m\left(2^{-j} \theta\right)=m_{0}(\theta)$ so that $2^{-j} m^{\prime}\left(2^{-j} \theta\right)=m_{0}^{\prime}(\theta)$ and

$$
T_{j} f(x)=\int_{\pi / 8}^{\pi / 4} m_{0}^{\prime}(t) H_{2^{-j}} S_{j} f(x) d t .
$$

Now

$$
\begin{aligned}
\left\|\left(\sum_{j}\left|T_{j} f\right|^{2}\right)^{1 / 2}\right\|_{p} & \leq \int_{\pi / 8}^{\pi / 4}\left|m_{0}^{\prime}(t)\right|\left\|\left(\sum_{j}\left|H_{2^{-j}} S_{j} f\right|^{2}\right)^{1 / 2}\right\|_{p} d t \\
& \leq C \int_{\pi / 8}^{\pi / 4}\left|m_{0}^{\prime}(t)\right| d t\|f\|_{p}
\end{aligned}
$$

using (1) as in the case $q=2$ of the preceding section. This ends the proof of Theorem 2.

From the proof of this theorem it is immediate that for a general $m$ (that is, not necessarily obtained by dilating a fixed one) the hypothesis

$$
\int_{\pi / 8}^{\pi / 4} \sup _{j} 2^{-j}\left|m^{\prime}\left(2^{-j} t\right)\right| d t<+\infty
$$

also gives an $L^{p}$-multiplier, $1<p<\infty$.

\section{HÖRMANDER-TYPE THEOREMS}

Let $J_{\alpha}$ be the Bessel potential of order $\alpha$ given by

$$
J_{\alpha} f(\theta)=\sum_{k}\left(1+|k|^{2}\right)^{\alpha / 2} \hat{f}(k) e^{i k \theta}
$$

for $f$ defined on $S^{1}$. The Bessel potential spaces are defined as

$$
\mathscr{L}_{\alpha}^{q}\left(S^{1}\right)=\left\{f \in L^{q}\left(S^{1}\right) \text { s.t. } J_{\alpha} f \in L^{q}\left(S^{1}\right)\right\} .
$$

When $\alpha$ is an integer this condition is equivalent to $D^{j} f \in L^{q}, 0 \leq j \leq \alpha$ and $\mathscr{L}_{\alpha}^{q}$ coincides with a Sobolev space. See [6] for details.

If $m_{0}$ is compactly supported on $(\pi / 8, \pi / 4)$ and belongs to $\mathscr{L}_{1}^{q}\left(S^{1}\right)$ for some $q>1$ (so that it is bounded as a consequence of the embedding theorems) then Theorem 2 is applicable. If we merely assume that $m_{0}$ is bounded then the consequence of the theorem holds only in $L^{2}$. By interpolation we get

Corollary 4. Let $m_{0} \in \mathscr{L}_{\alpha}^{q}\left(S^{1}\right)$ for some $q>1, \frac{1}{q}<\alpha \leq 1$, supported on $(\pi / 8, \pi / 4)$, then $m(\theta)=\sum_{j} m_{0}\left(2^{j} \theta\right)$ is in $\mathscr{M}_{p}$ for

$$
\left|\frac{1}{p}-\frac{1}{2}\right|<\frac{\alpha}{2}
$$


In a similar way we obtain a corollary to Theorem 1 . Let $\psi$ be a positive $C^{\infty}$ function supported on $(\pi / 8, \pi / 2)$ such that

$$
\sum_{j=0}^{\infty} \psi\left(2^{-j} \theta\right) \equiv 1 \text { on }\left(0, \frac{\pi}{4}\right)
$$

then

$$
\sup _{j}\left\|m\left(2^{j} \cdot\right) \psi\right\|_{\mathscr{L}_{1}^{q}}<+\infty
$$

implies condition (1) for the same $q$. Thus we have

Corollary 5. With the preceding notation, assume that $m$ verifies

$$
\sup _{j}\left\|m\left(2^{j} \cdot\right) \psi\right\|_{\mathscr{L}_{\alpha}^{q}}<\infty .
$$

Then, $m$ is in $\mathscr{M}_{p}$ for:

(i) $\left|\frac{1}{p}-\frac{1}{2}\right|<\frac{3 \alpha}{4}-\frac{1}{2 q}$ if $\frac{1}{q}<\alpha \leq \min \left(\frac{2}{q}, 1\right)$,

(ii) $\left|\frac{1}{p}-\frac{1}{2}\right|<\frac{\alpha}{2}$ if $\frac{2}{q}<\alpha \leq 1$.

The presence of the supremum in the condition makes the interpolation more difficult now. But it still can be done by using Calderón's upper $s$ method (see [2]).

\section{FINAL REMARKS}

We do not know whether Theorem 1 and Corollary 5 are sharp or not. An alternative approach using weighted inequalities for homogeneous singular integrals gives us the same results. We will not develop this method here because the proof becomes longer than the one we gave above. Just a few comments about it.

The inverse Fourier transform of $m \in \mathscr{L}_{1}^{q}, 1<q<\infty$, is the sum of a multiple of the Dirac delta and a principal value distribution, p.v. $\Omega(x)|x|^{-2}$, where $\Omega$ is homogeneous of degree zero, its restriction to $S^{1}$ has mean value zero and is in $L^{q}\left(S^{1}\right)$.

Convolution with p.v. $\Omega(x)|x|^{-2}$ is bounded in the space $L^{2}(w)$ with weight $w$ depending on $\Omega$ in general. When $\Omega$ belongs to $L^{2}\left(S^{1}\right)$ this $w$ can be taken in the Muckenhoupt $A_{1}\left(\mathbb{R}^{2}\right)$ class and inequality (5) holds uniformly with $U(x)=\left(M_{S} u^{\delta}(x)\right)^{1 / \delta}$. The strong Hardy-Littlewood maximal function appears instead of the usual maximal function because the dilation in the angle $m\left(2^{j} \theta\right)$ is essentially equivalent to the change of variables $\xi_{1} \rightarrow 2^{j / 2} \xi_{1}, \xi_{2} \rightarrow 2^{-j / 2} \xi_{2}$ due to the homogeneity of $m$.

On the other hand, if $\Omega \in L^{q}, 1<q<2$, and one tries to avoid dependance on $\Omega$ (which is necessary in Theorem 1 and not in Theorem 2) the class of weights is restricted to those satisfying $M_{\Omega^{2-q}} w \leq C w$ a.e. for all $\Omega \in L^{q}$ where

$$
M_{\Omega} w(x)=\sup _{h>0} \frac{1}{h^{2}} \int_{B(0, h)}|\Omega(y) \| w(x-y)| d y .
$$


This restriction implies the one appearing in inequality (4).

Weighted norm inequalities for homogeneous singular integrals of the type cited above can be seen in [3].

\section{ACKNOWLEDGMENT}

José Luis Rubio de Francia, our friend and teacher, died in February 1988. It has been a great honor to have worked with him and it is a pleasure to see how the methods he developed are present in this paper.

We are grateful to A. Carbery for very interesting discussions and valuable suggestions on the results of this paper.

\section{REFERENCES}

1. M. Christ, J. Duoandikoetxea, and J. L. Rubio de Francia, Maximal operators related to the Radon transform and the Calderon-Zygmund method of rotations, Duke Math. J. 53 (1986), 189-209.

2. W. C. Connet and A. L. Schwartz, A remark about Calderón's upper s method of interpolation (Proc. of the Conf. on Interpolation Spaces and Allied Topics in Analysis), Lecture Notes in Math., vol. 1070, Springer-Verlag, 1984.

3. J. Duoandikoetxea, Weighted norm inequalities for homogeneous singular integrals, preprint.

4. J. Garcia-Cuerva and J. L. Rubio de Francia, Weighted norm inequalities and related topics, North-Holland, Amsterdam, 1985.

5. A. Nagel, E. M. Stein, and S. Wainger, Differentiation in lacunary directions, Proc. Nat. Acad. Sci. U.S.A. 75 (1978), 1060-1062.

6. E. M. Stein, Singular integrals and differentiability properties of functions, Princeton Univ. Press, Princeton, NJ, 1970.

Departamento de Matemáticas, Universidad Autónoma, 28049 Madrid, Spain

Departamento de Matemáticas, Universidad del Pais Vasco-Euskal Herriko Unibertsitatea, Apartado 644, 48080 Bilbao, Spain 